University of Wollongong

Research Online

Faculty of Social Sciences - Papers (Archive) Faculty of Arts, Social Sciences \& Humanities

2010

Characteristics of the closest relationships between children with developmental disabilities and peers in inclusive settings

Amanda A. Webster

University of Wollongong, awebster@uow.edu.au

Mark Carter

Macquarie University

Follow this and additional works at: https://ro.uow.edu.au/sspapers

Part of the Education Commons, and the Social and Behavioral Sciences Commons

Research Online is the open access institutional repository for the University of Wollongong. For further information contact the UOW Library: research-pubs@uow.edu.au 


\title{
Characteristics of the closest relationships between children with developmental disabilities and peers in inclusive settings
}

\begin{abstract}
Inclusive education has become more common in schools, and children with developmental disabilities have had greater opportunities to interact, and hopefully establish relationships with their typically developing peers. While the quality of friendships between typically developing children has been examined in detail, relatively little comparable data is available on children with developmental disabilities. The current study provided an examination of the characteristics of the closest relationships between children with developmental disabilities and peers in inclusive school settings. Twenty-five children with developmental disabilities aged between approximately 5 and 12 years participated. Using an interview instrument, the relationships of these children with 74 peers were examined across six dimensions. Overall, dyads were found to be high in Validation and Caring as well as Help and Guidance, followed by slightly lower levels of Companionship. Intimate Exchange was reported to be lower. Conflict among dyads was also low, and Conflict Resolution was reported to be high when problems did occur. There was a clear differentiation between the highest- and lowest-ranked dyads for children with a disability. Overall, the features of the relationships between children with disabilities and their highestranked peer appeared similar in nature to those previously reported between typically developing peers.
\end{abstract}

\section{Keywords}

developmental, closest, characteristics, children, inclusive, between, peers, disabilities, settings, relationships

\section{Disciplines}

Education | Social and Behavioral Sciences

\section{Publication Details}

Webster, A. A. \& Carter, M. (2010). Characteristics of the closest relationships between children with developmental disabilities and peers in inclusive settings. Australasian Journal of Special Education, 34 (1), 61-78. 


\title{
Characteristics of the Closest Relationships Between Children With Developmental Disabilities and Peers in Inclusive Settings
}

\author{
Amanda A. Webster and Mark Carter \\ Macquarie University Special Education Centre, Sydney, Australia
}

\begin{abstract}
Inclusive education has become more common in schools, and children with developmental disabilities have had greater opportunities to interact, and hopefully establish relationships with their typically developing peers. While the quality of friendships between typically developing children has been examined in detail, relatively little comparable data is available on children with developmental disabilities. The current study provided an examination of the characteristics of the closest relationships between children with developmental disabilities and peers in inclusive school settings. Twenty-five children with developmental disabilities aged between approximately 5 and 12 years participated. Using an interview instrument, the relationships of these children with 74 peers were examined across six dimensions. Overall, dyads were found to be high in Validation and Caring as well as Help and Guidance, followed by slightly lower levels of Companionship. Intimate Exchange was reported to be lower. Conflict among dyads was also low, and Conflict Resolution was reported to be high when problems did occur. There was a clear differentiation between the highest- and lowest-ranked dyads for children with a disability. Overall, the features of the relationships between children with disabilities and their highestranked peer appeared similar in nature to those previously reported between typically developing peers.
\end{abstract}

Keywords: friendship, relationships, children, developmental disabilities, peers

The past two decades have seen an important shift in educational practice for children with disabilities so that even children with severe disabilities are being included and provided with instruction in regular education settings (Falvey, Givner, \& Kimm, 1995; Moore, 1998; Smith, 1998; TASH, 1999). The development of relationships with peers has been identified as one of the most important outcomes of these inclusive practices (TASH, 1999). A significant body of research suggests children with developmental disabilities are at high risk of experiencing difficulties with social integration (Barber \& Hupp, 1993; Bauminger \& Kasari, 2000; Fujiki,

Address for correspondence: Amanda Webster, Macquarie University Special Education Centre, Macquarie University NSW 2109, Australia. E-mail: Seapig@octa4.net.au 
Brinton, Hart, \& Fitzgerald, 1999; Guralnick, 1997; Guralnick \& Groom, 1988; Hanline, 1993; Hurley-Geffner, 1995; Strain, 1984), and thus, the relationships and friendships formed by this group in inclusive school settings is of particular interest.

Extensive research has been conducted to investigate aspects of relationships and friendships among typically developing children (e.g., Berndt, 1981; Berndt \& Perry, 1986; Bigelow, 1977; Cleary, Ray, LoBello, \& Zachar, 2002; Furman \& Bierman, 1983; Parker \& Asher, 1993; Ray \& Cohen, 1996). In the extant literature, friendship is generally viewed as a relationship between two individuals that is characterised by the features of shared interaction, mutual liking, and mutual enjoyment. As early as 1983, Gottman (1983) documented the formation of friendships and explored basic processes that transpired during the development of these relationships. More recent literature has provided descriptions of the developmental patterns and general dimensions (such as Conflict, Help and Companionship) that occur in relationships between children at different stages. Two definitive studies were conducted by Parker and Asher (1993) and Bukowski, Hoza, and Boivin (1994) in which the researchers use interviews to describe the relationships of children across similar dimensions. These interviews have formed the basis of much of the following research (e.g., Chamberlain, Kasari, \& Rotherham-Fuller, 2007; Wiener \& Schneider, 2002) on children's relationships.

To date, much of the research examining the social relationships or friendships of children with disabilities has not actually focused on the features of these relationships, as has been the case with typically developing children. For example, some researchers have explored the impact that different environments have had on the development of relationships between children with disabilities and typically developing peers (Buysse, Goldman, \& Skinner, 2002; Chamberlain et al., 2007). Others (e.g., Guralnick, 1997; Guralnick \& Groom, 1988; Strain, 1984) have investigated the effects of individual characteristics, such as level of disability, or have examined behaviours of children with disabilities (Bauminger \& Kasari, 2000; Buysse, 1993; Field, 1984; Guralnick \& Groom, 1988; Hall, 1994; Hall \& Strickett, 2002) to determine whether they correlated with the development of relationships and friendships. Other researchers (Bauminger \& Kasari, 2000) have examined specific behaviours, such as the tendency of children with autism to engage in activities with peers that provide limited opportunities for interaction, thereby restricting options for the development of meaningful relationships. Although this body of research examines some children's behaviours, it does not fundamentally provide information on the specific dimensions that may characterise the relationships between children with disabilities and peers.

Some researchers have focused on collecting data on perceptions of others regarding important dimensions of relationships. Although these studies have provided some description of the dimensions in relationships (Buysse, Nabors, Skinner, \& Keyes, 1997; Hendrickson, Shokoohi-Yekta, Hamre-Nietupski, \& Gable, 1996), they are limited by the exclusive measurement of perceptions of third parties (e.g., teachers). Another area of research has focused on the measurement of behaviours associated with time-limited and finite interactions between children in order to evaluate the status of their relationships and friendships (e.g., Evans, Salisbury, Palombaro, Berryman, \& Hollowood, 1992; Freeman \& Kasari, 2002; Lee, Yoo, \& Bak, 2003; Siperstein, Leffert, \& Wenz-Gross, 1997). One limitation of many of these studies, however, is that they have examined interactions over very short periods of time that did not necessarily occur within the context of a sustained relationship. In studies (e.g., Evans et al., 1992) where a relationship was assumed between students, the limited time-span of observations is problematic as it may or may not have reflected the true nature of the relationship (Meyer et al., 1998; Staub, Schwartz, Gallucci, \& Peck, 1994). 
Ultimately, these studies offer information on interactions in a restricted time period and setting and give only very limited insights into dimensions of the relationships or friendships between children with developmental disabilities and their peers. A small number of studies (Cuckle \& Wilson, 2002; Heiman, 2000; Kennedy \& Itkonen, 1994; Zetlin \& Murtaugh, 1988) have used more direct methods to ascertain some general features that may be indicative of the nature of relationships between children with disabilities and their peers. None of these studies, however, specifically examined relationships of children in inclusive settings. They also addressed only a small number of general dimensions in relationships between children with developmental disabilities and typically developing peers.

A body of research that offers preliminary insights into some of the possible dimensions of the relationships that children with developmental disabilities form with their peers includes a group of qualitative studies conducted in a variety of inclusive and specialised school settings (Murray-Seegert, 1989; Salisbury \& Palombaro, 1998; Strully \& Strully, 1985). While providing a valuable understanding of these relationships, the authors of these studies acknowledge that their research was somewhat limited by factors such as sample size and lack of generalisability and advise that the results be used as a preliminary description of the relationships between children with disabilities and typically developing peers (Meyer et al., 1998; Richardson \& Schwartz, 1998; Salisbury \& Palombaro, 1998; Staub, 1998).

There have been few attempts to apply a systematic empirical approach to describe the relationships or friendships of children with disabilities. Wiener and Schneider (2002) provided an illustration of the use of questionnaire-based methodology to describe the friendships of a group of children, but their focus was on learning disabilities rather than intellectual or developmental disabilities. A recent study conducted by Chamberlain et al. (2007) did use the Friendship Quality Scale (Bukowski et al., 1994) to investigate relationships and friendships between 17 children with autism or Asperger's syndrome. The researchers in this study, however, compared the perceptions of target students with those of their peers regarding the quality of the relationship and were not concerned with describing these relationships according to the dimensions addressed in the interview instrument. Bauminger and Kasari (2000) also use the Friendship Quality Scale in a study conducted with children with high functioning autism. As the focus of this study was primarily to examine loneliness in these children, only limited results on dimensions of the children's relationships were presented.

Recently, Webster and Carter (in press) have described adaptations to the Friendship Quality Questionnaire (Parker \& Asher, 1993) to allow its application to students with more significant cognitive and communication impairments. These adaptations included a simplified response scale, procedural modifications, creation of an alternative short interview form and the use of multiple respondents to obtain a more complete dataset. These modifications were successful in obtaining reliable responses from a wide range of children and provided information on children's relationships that might not have been obtained using the traditional interview instrument. Thus, these adaptations open the way for detailed examination of the characteristics of relationships in children with more significant disabilities in inclusive school settings.

Studies on children with developmental disabilities have examined the closest relationships of these children across a variety of settings. As educating students with developmental disabilities in inclusive settings is now considered the preferred educational option in many school communities (Coots, Bishop, Grenot-Scheyer, \& Falvey, 1995; Falvey, 1995; Westling \& Fox, 2004), the investigation of relationships in inclusive settings is 
imperative. Although quite a few studies have focused on comparisons between relationships of children in inclusive as opposed to specialised settings (Buysse \& Bailey, 1993; Buysse et al., 2002; Freeman, 2000; Heiman, 2000), few studies have investigated the relationships of children who have all been exposed to inclusive settings for several years. In addition, the majority of research has focused on middle class and Caucasian populations and has primarily been conducted in cities in North America or Great Britain. In order to gain a fuller understanding of the social relationships of children with disabilities as a group, it is important that investigations be conducted in varied settings and with populations that are more diverse.

Variations on the Friendship Quality Scale that was originally developed by Bukowski et al. (1994) (e.g., Asher, Parker, \& Walker, 1996; Ladd, Kochenderfer, \& Coleman, 1996; Newcomb \& Bagwell, 1996; Parker \& Asher, 1993) have provided a rich and detailed insight into the nature of relationships and friendships between typically developing children. This methodology has been applied to children with learning disabilities (Wiener \& Schneider, 2002) and to a limited extent to children with high functioning autism (Bauminger \& Kasari, 2000; Chamberlain et al., 2007). Very limited research, however, has been conducted with children with more significant levels of disability, specifically those with developmental disabilities, who are increasingly the focus of inclusion in schools and are at significant risk of experiencing difficulties with social integration. The study reported in this article was part of a larger project that examined relationships including friendships between children with developmental disabilities and peers in inclusive school settings. Procedural adaptations described by Webster and Carter (in press) were used with a version of the Friendship Quality Scale to attempt to describe and characterise the dimensions of the closest relationships between children with developmental disabilities and peers identified as friends in inclusive settings.

\section{Methodology}

\section{Setting}

The research was conducted in Alice Springs, Australia. Alice Springs has a population of approximately 27,000 people, which includes an estimated 5000 Aboriginal Australians. Due to various employment and lifestyle opportunities, Alice Springs has a diverse population with immigrants from many countries and cultures. It is located at the centre of Australia and is $1300 \mathrm{~km}$ from any city with a larger population.

\section{Selection of Target Students}

A letter was sent to all area primary schools detailing the basic parameters of the study and outlining the criteria for selection of the target students. Schools were asked to identify any student who: (1) had been identified as having a developmental disability, which was defined as a significant delay in adaptive behaviour and at least one other area of functional impairment such as cognitive or communication skills (Centre for Developmental Disability Studies, 2001; Developmental Disabilities Assistance and Bill of Rights Act 2000; National Association of Developmental Disabilities Councils, 2003; Northern Territory Government, 2005); (2) had a high level of educational need in that he/she had been identified by the Northern Territory Department of Education, Employment and Training as in need of individual assistance in order to access the curriculum; (3) had a record of regular attendance and/or would be present in school for the entire school year; (4) had not been identified as a child whose primary disability was a sensory impairment (i.e., impairments in hearing, vision, physical skills), or 
behaviour problems. It should be noted that these needs may have existed as a secondary issue to the developmental disability. Children were excluded from the study if the primary diagnosis was a learning disability with average intellectual abilities, low achievement, and no corresponding significant delays in other areas or adaptive behaviour.

All ten area public and private primary schools agreed to participate in the study, but three private schools (two of which were very small) reported that they did not have any students who met the specified criteria. Participating schools nominated all students who met the criteria for the study. Parental consent was obtained and the names of nominated students were submitted to the researchers. Twenty-nine students were initially nominated. Another student was added by a school at a slightly later date. It should be noted that although the Northern Territory has a general policy of including children with significant disabilities, Alice Springs does have one special school for children with severe to profound multiple disabilities. The majority of these students have come from remote communities. At the time of the study, only five students permanently residing in Alice Springs attended the special school. The 30 nominated students represented the entire population of eligible students attending regular schools in Alice Springs. Schools were not able to contact the parents of four students and one withdrew after the initial assessment. Thus, parental consent was obtained for 25 students. These children represented $83 \%$ of those with developmental disabilities in inclusive schools in Alice Springs.

\section{Target Students}

Six of the selected students were in preschool (mean age $=5: 4$, range 4:9-6:1), 12 were in the junior primary grades of transition through Year 3 (mean age $=7: 2$, range 5:19:4), and seven were in senior primary Grades Year 4 through 6 (mean age $=10: 9$, range 10:0-12:1). Target students were predominately male with four girls and 21 boys. It should be noted that males tend to predominate in prevalence figures for developmental disabilities (Bhasin, Brocksen, Avchen, \& Braun, 2006; Rice, 2007) and more importantly, that this ratio was the true representation of boys to girls in the target population in the Alice Springs area. Eight children were identified as being of Aboriginal descent. Two of these students spoke a language other than English at home. Based on diagnostic reports of the 25 target students with a developmental disability, 13 students had a primary diagnosis of an intellectual disability (eight mild, four moderate, and one severe) with compounding disabilities in communication and motor skills. Six students had a primary diagnosis of an autistic spectrum disorder, one student had cerebral palsy, two students had severe communication disorders with compounding social-emotional and learning delays, and three students had unspecified developmental delays with deficits in multiple areas.

The Vineland Adaptive Behavior Interview (Sparrow, Balla, \& Cicchetti, 1985) and the Social Skills Rating System (Gresham \& Elliott, 1990) were completed for each student using the teacher as an informant. The mean score for the Vineland Adaptive Behavior Composite was 64.6 (range 42-78). In addition, 19 students (76\%) had a composite score that was less than 70 while four students $(16 \%)$ had scores that were below 60. Assessment on the Social Skills Rating System (Gresham \& Elliott, 1990) produced a group mean standard score of 77.7 (range 54-97) on the Social Skills subtest. This is in the low range and reflects fewer social skills than would be expected for students in either the preschool or primary school age groups. A group mean standard score of 113 (range 88-137) was found across the 25 target students on the 
Problem Behavior subtest. This score falls at the high end of the average range. It is important to note that a higher score indicates the child exhibits a higher number of problematic behaviours that may interfere with learning. For students in the primary age group, a mean standard score of 80.3 (range 70-106) was found for the academic measure. This score falls within the 'below' range when compared to the norm.

\section{Nomination of Peers}

As some researchers (Hurley-Geffner, 1995; Siperstein et al., 1997) have suggested that children with disabilities may have relationships that are different to those of typically developing peers, it was determined that peer selection would not be limited by sex, age, or gender, but would be restricted only to peers in the school setting. Nominations were not limited to the child's class as children may have formed a relationship or friendship with a peer in a subsequent year but had been placed in a corresponding class in the current year. Thus, sociometric analysis was not considered to be appropriate. Further, previous researchers (Gest, Graham-Bermann, \& Hartup, 2001) have questioned the validity of using sociometric instruments to identify friends, indicating these measures may reflect personal popularity rather than suggesting friendship per se. Relatives or other children with disabilities were also not specifically excluded. This decision was made because past researchers have demonstrated that relatives play a significant part in the social relationships of children from specific cultural groups, including children from Hispanic American (Turnbull, Blue-Banning, \& Pereira, 2000) and Aboriginal Australian (Searle, 1989) backgrounds. It should be noted that although not excluded, no siblings were nominated as peers and only one cousin was nominated.

The wide range of ages and communication levels of the target population in the present study, however, made it necessary to find a method of peer nomination that allowed those target students who were capable to participate in the peer selections. Thus, it was decided that a combination of teacher and target student nomination would be used. At the discretion of the teacher, older and more verbal students were asked to identify their three closest friends. In these instances, each student's choices were verified by his/her teacher. For the remaining target students, teachers were asked to nominate three friends or, if three friends could not be identified, children with whom the target student had the closest relationships or most frequently interacted. Target students were then asked if the three peers chosen by their teacher were their friends along with an additional three students selected randomly from their class as distractors. If the target student said the peers identified by the teacher were not his/her friends, the teacher was asked to identify another peer. This alternative selection was subsequently confirmed by asking the target student whether the nominated peer was a friend. The target student was again asked about the nominated peer as well as a randomly selected peer who served as a distractor. All target students confirmed teachers' selections of peers with the exception of five who were nonverbal.

All nominated peers participated in an interview concerning their relationship with the target student. During these interviews, peers were also asked to nominate their friends. Thirty-four peers identified the target student as a friend when asked to list their friends at the beginning of the interview. If the peer did not voluntarily nominate the target child, he/she was asked a direct question as to whether the target student was a friend. Twenty-seven peers confirmed that the target student was his/her friend after being directly asked, and 12 peers stated that the target student 
was not a friend when asked. One peer was not able to confirm or deny the target student as a friend due to limited proficiency in English. Peers were interviewed regardless of whether they indicated the target student was a friend.

Seventy-four peers were selected for the 25 target students as one nominated peer moved during the initial phase of the study. Twenty-two peers were female and 52 were male. Fourteen nominated peers were of Aboriginal heritage. Seven nominated peers were identified as having English as their second language. Eleven nominated peers were currently enrolled in classes other than the ones in which the target students were enrolled. Five nominated peers were selected by more than one target student and two target students were also selected as nominated peers by other target students. As far as the researchers were able to ascertain, 11 additional nominated peers had also been identified as having some type of special need such as ADHD or a speech and language delay. These students, however, were not selected as target students as they did not meet the criteria for level or type of disability.

\section{Data Collection}

\section{Interviews}

Both the interview instrument and procedures have been described in detail by Webster and Carter (in press) and will be briefly overviewed here. A 48 item questionnaire (plus three repeated items to assess reliability) was adapted from instruments previously used by Parker and Asher (1993) and Bukowski et al. (1994). The instrument addressed the following dimensions of relationships: (1) Validation and Caring, (2) Conflict Resolution, (3) Conflict, (4) Help and Guidance, (5) Companionship, and (6) Intimate Exchange. Adaptations included procedural and response scale modifications (including a 'don't know' option), the use of multiple respondents in interview sessions, and the creation of an alternative short interview form for children who could not complete the full interview form. The short interview form was given to all students in preschool and transition classes ( 5 years) and to those target students whose teachers or parents indicated would not be able to complete the full interview form. The remaining target students, peers and all adults completed the long interview form. The 10 questions on the short interview form were also included as the first questions on the long interview form. Data were collected through interviews with the target students, nominated peers, teachers, and parents and were conducted at schools for all participants with the exception of some parents. The remaining parent interviews were conducted at community locations. Target students, parents, and teachers were asked each question three times in succession for each of the nominated peers. A 3-point scale with responses of 'always', 'sometimes' and 'never' was used with all respondents and a response option of 'I don't know' was provided. Parents and teachers were asked questions from the standpoint of the target student. Interview sessions lasted approximately 10 to 60 minutes depending on whether the short interview form or the full interview form was used. Except in one instance, interviews were administered in a single session. One nominated peer stated he was tired after 30 minutes and requested the interview be completed on the following day. Generally interview session with adults tended to last longer than those with target students or nominated peers.

Three of the first 10 questions were repeated for child respondents to assess reliability. Reliability of child respondents was very high and only one target student and one nominated peer were rejected because they did not meet the reliability criteria (100\% agreement) across three repeated questions (Webster \& Carter, in press). Sixty percent of target students, $89 \%$ of peers, and $100 \%$ of teachers and parents successfully 
completed the short interview form. Additionally $40 \%$ of target students, $61 \%$ of peers, as well as $100 \%$ of teachers and parents also completed the full interview form. High levels of reliability were found between short and long forms of the interview questionnaire (Webster \& Carter, in press).

\section{Data Analysis}

Prior to analysis, responses were converted into numerical scores ranging from 3 for Always, 2 Sometimes, and 1 for Never. A score for each relationship dimension was calculated for both the short and full forms of the interview. First, a separate score for each dyad was calculated for all four respondents (target child, peer, teacher, parent), who had a complete dataset across all questions relevant to a given dimension. This was accomplished by calculating the mean of the responses to all relevant questions. If an interviewee failed to respond or responded 'I don't know' to a relevant question, their data were excluded. To aid in sorting data into high and low scoring groups, a Mean Interview Score was then calculated by averaging the specific dimension score of all respondents who had a complete dataset for each dyad. Thus, the Mean Interview Score was based on between one and four respondent scores, depending on the amount of incomplete data. There was some concern about this approach in calculating the Mean Interview Score. Therefore, in order to evaluate the consistency of respondent scores for each dyad, an average deviation was calculated for all dyads with multiple respondent scores. A mean average deviation score of 0.30 ( $S D=0.06$, range $0.23-0.39$ ) was calculated across all dimensions in the short interview form and a mean average deviation of 0.25 ( $S D=0.05$, range $0.18-0.32$ ) was calculated across all dyads and dimensions in the full interview form indicating a fairly high degree of consistency across multiple respondent scores. Nevertheless, Help and Guidance (0.39) in the full interview form and Conflict Resolution (0.32) in both interview forms were above 0.30 and caution should be exercised in interpreting these data.

\section{Results}

\section{Dimensions of Relationships}

\section{Interviews}

Mean Interview Scores and standard deviations for each dimension across all dyads and respondents are presented in Table 1. Overall, findings for both analyses indicate that dyads reported the highest scores for Conflict Resolution and Validation and Caring, medium scores for Help and Guidance and Companionship, and generally lower scores for Conflict and Intimate Exchange. One interesting finding was that when dyads were grouped by Mean Interview Score, it was evident that dyads in the same group, whether high or low scoring, were often linked to the same target students. This was true for several dimensions including Companionship, Validation and Caring, and Conflict. For example, the scores for Validation and Caring indicated that one target student had all three nominated peer relationships in the group of dyads with the top third of scores while another eight target students had at least two relationships in the group of dyads with the top third of scores. Similarly, two target students had all three relationships in the group of dyads with the lowest scores, with another target student having two relationships in this group.

\section{Age Comparison}

Due to the wide range of ages represented in the study, it was of interest to determine how data varied across age level. Results for each dimension were also sorted into 
TABLE 1

Interview Scores

\begin{tabular}{|c|c|c|c|c|c|c|c|}
\hline & & Companionship & $\begin{array}{l}\text { Validation } \\
\text { and caring }\end{array}$ & $\begin{array}{l}\text { Help and } \\
\text { guidance }\end{array}$ & $\begin{array}{l}\text { Intimate } \\
\text { exchange }\end{array}$ & Conflict & $\begin{array}{l}\text { Conflict } \\
\text { resolution }\end{array}$ \\
\hline \multicolumn{8}{|l|}{ Overall } \\
\hline \multirow[t]{2}{*}{ Short } & $M$ & 1.68 & 2.17 & 2.09 & 1.33 & 1.42 & a \\
\hline & $S D$ & 0.40 & 0.46 & 0.43 & 0.40 & 0.42 & \\
\hline \multirow[t]{2}{*}{ Full } & $M$ & 1.75 & 2.12 & 1.78 & 1.48 & 1.60 & 2.05 \\
\hline & $S D$ & 0.42 & 0.58 & 0.47 & 0.45 & 0.37 & 0.52 \\
\hline \multicolumn{8}{|l|}{ Age group } \\
\hline \multirow{2}{*}{$\begin{array}{l}\text { Short-upper primary } \\
\text { grades } 4-6(N=26)\end{array}$} & $M$ & 1.78 & 2.3 & 2.14 & 1.43 & 1.41 & a \\
\hline & $S D$ & 0.46 & 0.46 & 0.39 & 0.46 & 0.42 & \\
\hline \multirow{2}{*}{$\begin{array}{l}\text { Short-lower primary } \\
\text { grades } T-3(N=30)\end{array}$} & $M$ & 1.75 & 2.26 & 2.18 & 1.33 & 1.46 & a \\
\hline & $S D$ & 0.37 & 0.40 & 0.46 & 0.35 & 0.48 & \\
\hline \multirow{2}{*}{$\begin{array}{l}\text { Short-preschool } \\
4 \text { years old }(N=18)\end{array}$} & $M$ & 1.44 & 1.85 & 1.88 & 1.18 & 1.35 & a \\
\hline & $S D$ & 0.22 & 0.43 & 0.39 & 0.34 & 0.32 & \\
\hline \multirow{2}{*}{$\begin{array}{l}\text { Full-upper primary } \\
\text { grades } 4-6(N=26)\end{array}$} & $M$ & 1.84 & 2.24 & 1.97 & 1.57 & 1.54 & 2.12 \\
\hline & $S D$ & 0.45 & 0.55 & 0.42 & 0.40 & 0.41 & 0.57 \\
\hline \multirow{2}{*}{$\begin{array}{l}\text { Full-lower primary } \\
\text { grades } T-3(N=30)\end{array}$} & $M$ & 1.91 & 2.32 & 1.89 & 1.64 & 1.62 & 2.1 \\
\hline & $S D$ & 0.32 & 0.42 & 0.38 & 0.45 & 0.30 & 0.47 \\
\hline \multirow{2}{*}{$\begin{array}{l}\text { Full-preschool } \\
4 \text { years old }(N=18)\end{array}$} & $M$ & 1.44 & 1.45 & 1.33 & 1.1 & 1.66 & 1.81 \\
\hline & $S D$ & 0.30 & 0.39 & 0.39 & 0.28 & 0.42 & 0.44 \\
\hline \multicolumn{8}{|l|}{ Reciprocation } \\
\hline \multirow{2}{*}{$\begin{array}{l}\text { Short-voluntary } \\
\text { reciprocated } \\
\text { dyads }(N=34)\end{array}$} & $M$ & 1.86 & 2.34 & 2.27 & 1.43 & 1.32 & a \\
\hline & $S D$ & 0.37 & 0.42 & 0.36 & 0.37 & 0.36 & \\
\hline \multirow{2}{*}{$\begin{array}{l}\text { Short-confirmed } \\
\text { reciprocated } \\
\text { dyads }(N=27)\end{array}$} & $M$ & 1.57 & 2.19 & 2.06 & 1.31 & 1.51 & a \\
\hline & $S D$ & 0.40 & 0.36 & 0.42 & 0.47 & 0.49 & \\
\hline \multirow{2}{*}{$\begin{array}{l}\text { Short-not } \\
\text { reciprocated } \\
\text { dyads }(N=12)\end{array}$} & $M$ & 1.45 & 1.69 & 1.73 & 1.10 & 1.47 & a \\
\hline & $S D$ & 0.25 & 0.45 & 0.38 & 0.16 & 0.42 & \\
\hline \multirow{2}{*}{$\begin{array}{l}\text { Full-voluntary } \\
\text { reciprocated } \\
\text { dyads }(N=34)\end{array}$} & $M$ & 1.96 & 2.31 & 1.96 & 1.59 & 1.50 & 2.15 \\
\hline & $S D$ & 0.38 & 0.49 & 0.40 & 0.40 & 0.29 & 0.51 \\
\hline \multirow{2}{*}{$\begin{array}{l}\text { Full-confirmed } \\
\text { reciprocated } \\
\text { dyads }(N=27)\end{array}$} & $M$ & 1.64 & 1.78 & 1.68 & 1.44 & 1.64 & 1.95 \\
\hline & $S D$ & 0.36 & 0.57 & 0.50 & 0.47 & 0.40 & 0.54 \\
\hline \multirow{2}{*}{$\begin{array}{l}\text { Full-not } \\
\text { reciprocated } \\
\text { dyads }(N=12)\end{array}$} & $M$ & 1.43 & 2.07 & 1.43 & 1.26 & 1.79 & 1.91 \\
\hline & $S D$ & 0.36 & 0.65 & 0.43 & 0.47 & 0.45 & 0.45 \\
\hline
\end{tabular}

Note: $N=$ the number of dyads examined. $T$ = the Transition grade level which is the first year of school in which children are 5 years of age. ${ }^{a}=$ No question was included for Conflict Resolution on the short interview form.

preschool, junior primary, and senior primary age groups. This analysis is presented in Table 1. Consistently, junior primary and senior primary groups had similar scores across all dimensions on both short and full interview forms. Results for the preschool age group, however, were lower on all dimensions except Conflict in which all three age groups had fairly similar scores.

\section{Peer Reciprocation}

As previously mentioned in the description of the peer nomination process, all nominated peers were asked to list their friends at the beginning of each interview 
session as a measure of reciprocation of friendship. It was therefore of interest to determine whether results for dimensions of relationships varied according to degree of reciprocation. Peer reciprocation was coded as voluntary when the peer included the target child when asked to list his or her friends, confirmed when the peer indicated a target child was a friend after being asked directly, and not reciprocated when the peer stated the target child was not a friend. Relevant data are presented in Table 1. Four target students had all three dyads in the Voluntary group and 14 target students had at least one dyad in the Voluntary group. No target student had all three dyads in the Not Reciprocated group while seven target students had no nominated peers in the Voluntary Reciprocated group. When dyads were sorted into three groups based on levels of reciprocation, the results indicate that higher levels of reciprocation generally corresponded with higher scores on almost all of the positive dimensions of relationships. Validation and Caring was the only positive dimension in the full interview form in which the group of dyads with the lowest level of reciprocation did not have the lowest mean score for this dimension, although the highest reciprocation group did have the highest score. Similarly, dyads with higher levels of reciprocation had correspondingly lower scores in the negative dimension of Conflict. All positive dimensions in the short interview form showed decreasing scores in correspondence with lower levels of reciprocation. Scores for Conflict in the short interview form increased between Voluntary Reciprocated and Confirmed Reciprocated dyads, and then decreased slightly for the Not Reciprocated group. This score was higher, however, than the score for Conflict for the Voluntary Reciprocated group.

\section{Highest and Lowest Peer Comparison}

Previous researchers (Bukowski et al., 1994; Parker \& Asher, 1993; Wiener \& Schneider, 2002) primarily described friendships of target students and only one peer who had been determined to be the target student's closest friend. In contrast, the current study described the relationships of the target student and the three peers with whom he/she had the closest relationship, which might constitute a friendship. It was therefore of interest to determine whether the scale was sensitive enough to detect significant differences in the relationships between target students and each peer. Scores in the short interview form for each dimension were examined for the three dyads associated with each target student to determine which dyad had the most high or low scores across the five positive dimensions. The dyad for each target student who had the greatest number of high scores across the positive dimensions was designated the highest peer dyad. Similarly, the dyad who had the greatest number of low scores across the positive dimensions was designated the lowest peer dyad for each target student. It should be noted that scores for Conflict were not considered in the calculation of highest and lowest peer dyads as research has found that positive and negative aspects of relationships are not necessarily inversely correlated (Berndt, 2004). Thus dyads with high scores in positive dimensions would not necessarily be expected to have high scores for Conflict. All scores for highest peers dyads were compared to scores for lowest peer dyads and the results of this comparison are presented in Figure 1 for the short interview form and Figure 2 for the full interview form. Paired $t$ tests were conducted to determine if the difference in short interview form results for each dimension was statistically significant for highest peer dyads when compared to dyads comprised of lowest peers. Results for Companionship $(t=-5.51, d f=24, p=.00001)$, Validation and Caring $(t=-3.69, d f=23, p=.001)$, Help and Guidance $(t=-7.53, d f=24$, $p=.00000009)$, and Intimate Exchange $(t=-3.92, d f=24, p=.0006)$, were found to 


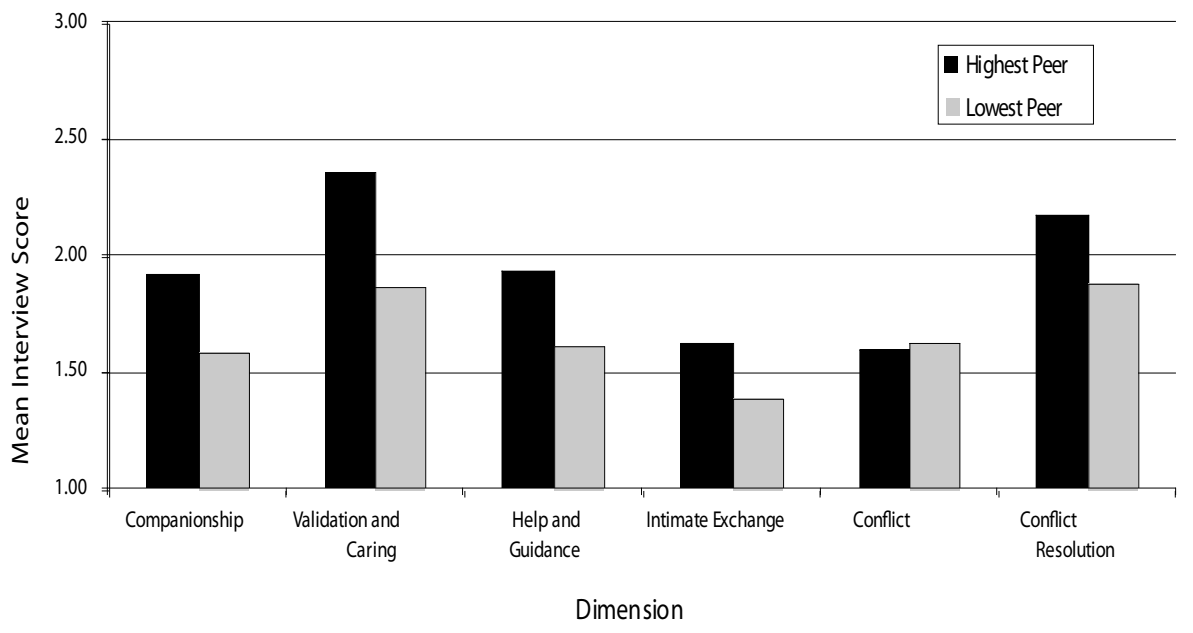

\section{FIGURE 1}

Features of relationships for highest and lowest peer dyads in full form.

be significant at the predetermined .05 level. Results for Conflict $(t=0.58, d f=24, p=$ .57 ) and Conflict Resolution $(t=-1.99, d f=19, p=.06)$ were not statistically significant. A similar pattern of difference was evident in the full interview form but inferential analysis was not conducted due to the incompleteness of data.

\section{Comparisons to Research on Typically Developing Children}

Researchers in previous studies (Bukowski et al., 1994; Bukowski, Hoza, \& Newcomb, 1987; Bukowski, Newcomb, \& Hartup, 1996; Parker \& Asher, 1993) have found similarities in the perceptions of dimensions in the relationships of typically developing children. Parker and Asher (1993) provided data on typically developing children that was comparable to a subset of data in the present study. Parker and Asher asked children to nominate their closest friend, thus only the highest ranked peer dyad, who could be

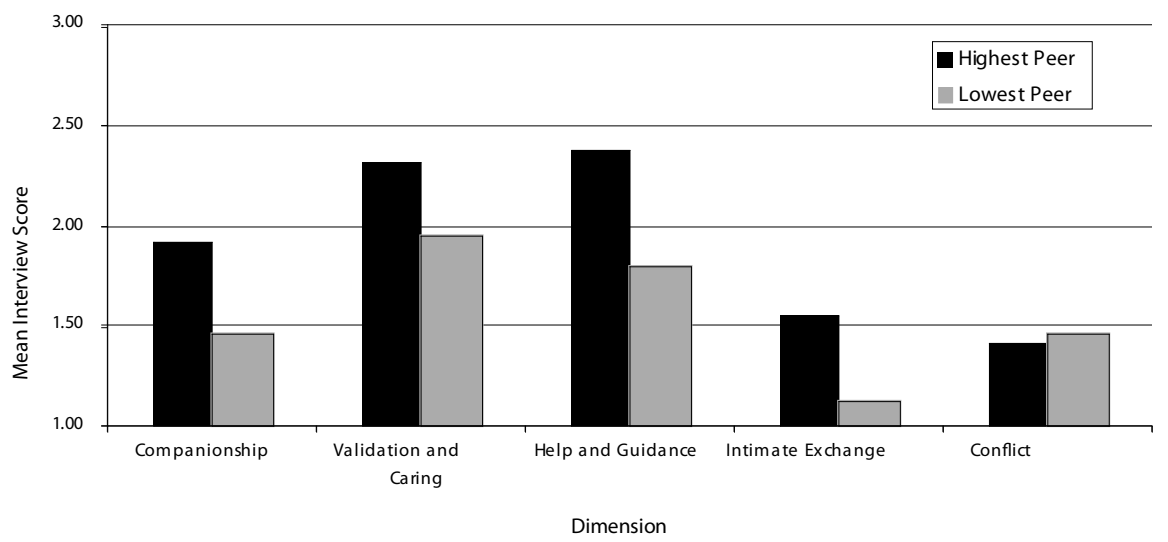

FIGURE 2

Features of relationships for highest and lowest peer dyads in short form. 
viewed as having the closest relationship for each student from the current study, was selected for comparison. The Mean Interview Scores were calculated for each target student and highest scoring peer for each of the six dimensions for the full interview form. Only the 12 target students in Grades 2 through 5, that were equivalent to the population included in the Parker and Asher study, were included in this analysis. These data along with the results from the Parker and Asher study are presented in Figure 3. While this was not series data, a line graph was selected as the best way to allow visual comparison of patterns across the dimensions. It should be noted that the interview data for the present study is plotted on a 3-point scale and is presented on the left side of the graph, whereas the Parker and Asher study use a 5-point scale, which is presented on the right side of the graph. As the scales are different, no comparisons of absolute values can be made, but the similarity of overall patterns can be examined. Although the scales employed in the two studies were different, the interview instruments utilized were almost identical.

The data in Figure 3 indicates that the overall patterns for the highest nominated peer according to peer, teacher, and mean interview scores are very similar to those found by Parker and Asher (1993). Although teacher scores decreased slightly more than the other three scores for Intimate Exchange and then increased somewhat for Conflict, the pattern demonstrated across dimension scores for peers and mean interview scores are similar to those presented by Parker and Asher. Thus, relationships between target children and the peer most consistently rated highly on all dimensions were reported to have similar features to the relationships of typically developing children in the Parker and Asher (1993) study.

\section{Discussion}

The present study was conducted in order to describe the closest relationships between children with developmental disabilities and their typically developing peers in inclusive

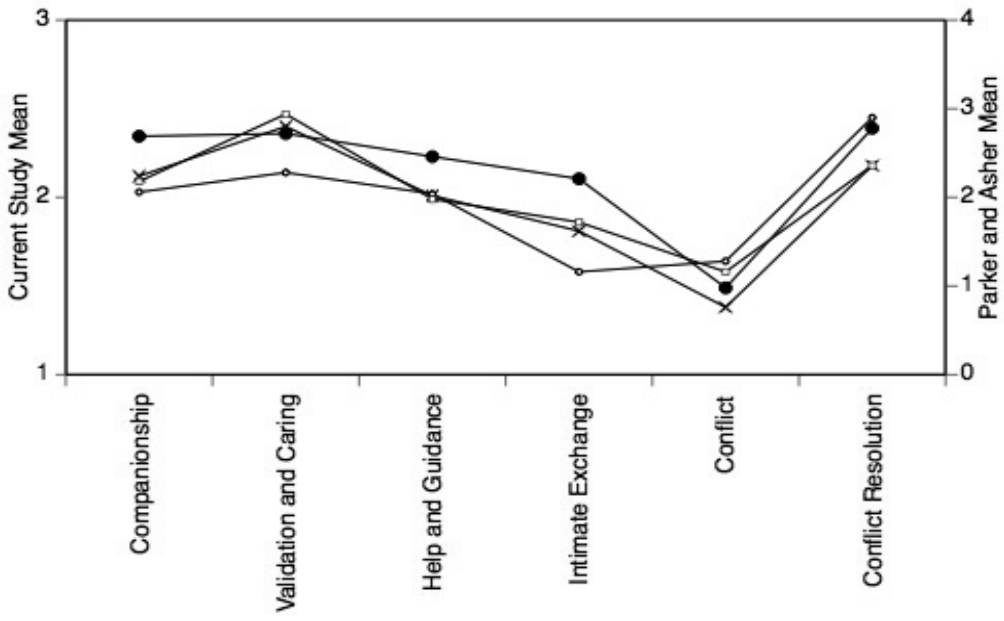

$$
\text { * Peer } \quad \rightarrow \text { Teacher } \quad \rightarrow \text { Mean } \quad \rightarrow \text { Parker \& Asher }
$$

FIGURE 3

Comparisons of results for highest nominated peer and typically developing children. 
settings. An adapted interview protocol was used to obtain information on a range of dimensions that have been previously examined in relationships between typically developing children.

Overall, Validation and Caring was generally reported to be high by the interviewees. It was also reported that dyads would help each other if needed as reflected in high scores for Help and Guidance. Results for Companionship, Conflict, and Conflict Resolution were also fairly positive with interviewees reporting that many dyads played together at school and resolved conflicts that occurred during interactions. In general, scores for Intimate Exchange were relatively low, suggesting that overall, dyads reported that they did not share much personal or emotionally laden information with each other. This finding is consistent with the results of previous researchers (Zetlin \& Murtaugh, 1988) who found that children with disabilities tended to display lower levels of intimate behaviours with peers in lunchrooms than did typically developing children in the same setting. In addition, these low scores may reflect the limited communication skills of many of the target students as Intimate Exchange tends to rely on a higher level of communication skills. Nevertheless, a great deal of diversity was found across dyads in their reporting of behaviours associated with the dimensions of friendship. In addition, although nomination of peers was adapted to include a combined nomination of target students and teachers, peer reciprocation data suggests that the majority of peers voluntarily listed target students as their friends. In addition, data for peers who denied friendship with target students corroborates results of previous studies including Freeman and Kasari (2002), who found that a proportion of peers nominated as a friend by children with Downs syndrome denied this friendship.

Comparison of the highest and lowest scoring dyad for each target student revealed a statistically significant difference on most dimensions. Although it was not the purpose of this comparison to determine a 'best friend' among the three peers, it was interesting to note that interviews were able to clearly discriminate between the highest and lowest ranked peer dyads, even though all peers were nominated as friends or, in lieu of friendship, peers with whom the target student most frequently interacted and therefore had the closest relationship. Conflict was the only dimension without a marked difference in scores for high and low scoring dyads. This is consistent with previous research (Kerns, 2000) that suggests that conflict may not necessarily be negatively correlated with friendship in all dyads and may, in fact, be a fairly salient feature of a friendship, even in dyads who have had comparatively long lasting relationships.

Comparison across age groups revealed that scores for dyads in preschool were lower for all dimensions except Conflict, in which scores were similar to or higher than those found in the junior or senior primary dyads. Results for junior primary and senior primary groups, however, were fairly similar across all dimensions. Although it is not possible at this time to determine the reasons for the differences in the preschool group, it seems possible, if not likely, that this may reflect developmental differences in the nature of relationships. In addition, results for dimensions were also compared across dyads grouped by level of peer reciprocation of friendship. A particularly interesting finding was that mean scores for the individual dimensions tended to correspond with levels of peer reciprocation for those dimensions. This finding would support the assertion of Asher, Parker, and Walker (1996) that reciprocation must be considered in determining friendship and friendship quality between children.

Although the primary purpose of the study was to examine and describe the relationships of children with developmental disabilities, it was also of interest to compare the results across dimensions with previous research on relationships between 
typically developing children. Opportunistic comparisons were made with the study conducted by Parker and Asher (1993). When highest ranked dyads in the present research were used for comparison with the 'best friend' in the Parker and Asher research on typically developing children, a very similar pattern of scores across the six dimensions of friendship was evident. This finding appears to suggest that at least a proportion of dyads, as reflected by the highest ranked dyads in the current research, demonstrated features of their relationships that were similar to those found in the friendships between typically developing children. Generally speaking, this is an encouraging finding for children who are included in mainstream classes. This result contrasts with the findings of Siperstein, Leffert and Wenz-Gross (1997) who reported that relationships between typically developing children were generally different to those between a typically developing child and a child with a disability. This inconsistency may have been in part due to the different methodologies as the Sipperstein, Leffert, and Wenz-Gross study primarily examined behaviours of dyads in simulated play sessions.

\section{Limitations and Future Research}

There are a number of limitations of the present study and directions for future research that warrant discussion. One of the aims of the current study was to extend previous research, which has primarily focused on children in middleclass settings with relatively ethnically circumscribed populations. The population in the current study included children from diverse cultural and socioeconomic backgrounds but there remains further need for replication of the research with other populations. In addition, there were few females in the study sample, reflecting a limited number of females in the eligible population. This may have had particular implications for results for Intimate Exchange as previous researchers (Bigelow, 1977; Furman \& Buhrmester, 1985) have found that intimacy levels vary between males and females, with females generally reporting higher levels of intimate exchange in their relationships. It should be noted however, that much of the research conducted with children with disabilities includes a predominately male population (e.g., Guralnick, 1997; Hall, 1994; Hall \& McGregor, 2000). In the present study only limited opportunistic comparison could be made between the relationships between typically developing children, specifically with those described by Parker and Asher. In addition, it should be noted that even though the results of comparisons between the research in the current study and the findings of Parker and Asher do indicate that similar patterns were found for the relationships of highest ranked dyads, these results should be interpreted with caution due to the differing scales used in the two studies. Further research should be conducted to compare such relationships within the same settings. In addition, the current research was limited to the school setting. Examination of relationships in home and other settings should be considered by future researchers. Finally, apart from an analysis across different age groups, the diverse characteristics of the target sample made analysis based on individual or demographic characteristics unwarranted.

Previous researchers (Salisbury \& Palombaro, 1998) have found that some relationships may change and evolve over time. The present study reflected only a crosssectional analysis of relationships and thus a need remains for longitudinal research to determine how characteristics of relationships change. Furthermore, Hurley-Geffner (1995) and Meyer et al. (1998) have suggested that children with disabilities may develop atypical relationships that are not found between typically developing peers. While the present research provided evidence that the closest relationships between children with disabilities and peers had similar characteristics to the friendships between 
typically developing children, further research should be conducted to investigate a broader range of relationships, including those that may be atypical. Finally, in the present study an attempt was made to evaluate the quality of relationships by describing their characteristics. Previous researchers (Kerns, 2000; Parker \& Asher, 1993) have suggested that another measure of the quality of children's relationships may be whether the children involved are satisfied. Further research should be conducted to determine the degree of correspondence between measured characteristics of relationships and children's reported satisfaction.

\section{Conclusion}

The current study was conducted in order to describe the general dimensions of the relationships between children with developmental disabilities and their peers in inclusive school settings. Each dimension was measured through interviews with multiple respondents. Dyads were found to be generally high in Validation and Caring and Help and Guidance followed by slightly lower levels of Companionship. Conflict among dyads was fairly low and Conflict Resolution was reported to be high when problems did occur. The exchange of personal information between dyads as reflected in the Intimate Exchange dimension was reported to be much lower than for the other positively directed dimensions. When results for the dyads comprised of the target student and the peer most frequently rated highly across all dimensions was compared to the relationships described in the research on typically developing children, a similarity was noted in the general patterns across all dimensions The results from the present study suggest that children with developmental disabilities can establish friendships with peers that have similar character to those of typically developing children.

\section{References}

Asher, S.R., Parker, J.G., \& Walker, D.L. (1996). Distinguishing friendship from acceptance: Implications for intervention and assessment. In W.M. Bukowski, A.F. Newcomb, \& W.W. Hartup (Eds.), The company they keep: Friendship in childhood and adolescence (pp. 366-405). New York: Cambridge University Press.

Barber, D., \& Hupp, S.C. (1993). A comparison of friendship patterns of individuals with developmental disabilities. Education and Training in Mental Retardation and Developmental Disabilities, 28, 13-22.

Bauminger, N., \& Kasari, C. (2000). Loneliness and friendship in high-functioning children with autism. Child Development, 71, 447-456.

Berndt, T.J. (1981). Age changes and changes over time in prosocial intentions and behavior between friends. Developmental Psychology, 17, 408-416.

Berndt, T.J. (2004). Children's friendships: Shifts over a half-century in perspectives on their development and their effects. Merrill-Palmer Quarterly, 50, 206-223.

Berndt, T.J., \& Perry, T.J. (1986). Children's perceptions of friendships as supportive relationships. Developmental Psychology, 22, 640-648.

Bhasin, T.K., Brocksen, S., Avchen, R.N., \& Braun, K.V.N. (2006). Prevalence of Four Developmental Disabilities Among Children Aged 8 Years - Metropolitan Atlanta Developmental Disabilities Surveillance Program, 1996 and 2000. Retrieved 10 July, 2008, from http://www.cdc.gov/mmwR/ preview/mmwrhtml/ss5501a1.htm\#tab1

Bigelow, B.J. (1977). Children's friendship expectations: A cognitive-developmental study. Child Development, 48, 246-253.

Bukowski, W.M., Hoza, B., \& Boivin, M. (1994). Measuring friendship quality during pre and early adolescence: The development and psychometric properties of the friendship qualities scale. Journal of Social and Personal Relationships, 11, 471-484. 
Bukowski, W.M., Hoza, B., \& Newcomb, A.F. (1987). Friendship, popularity, and the "self" during adolescence. Unpublished manuscript, University of Maine, Department of Psychology.

Bukowski, W.M., Newcomb, A.F., \& Hartup, W.W. (1996). Friendship and its significance in childhood and adolescence: Introduction and comment. In W.M. Bukowski, A.F. Newcomb, \& W.W. Hartup (Eds.), The company they keep: Friendship in childhood and adolescence (pp. 1-15). New York: Cambridge University Press.

Buysse, V. (1993). Friendships of preschoolers with disabilities in community-based child care settings. Journal of Early Intervention, 17, 380-395.

Buysse, V., \& Bailey, D.B., Jr. (1993). Behavioral and developmental outcomes in young children with disabilities in integrated and segregated settings. The Journal of Special Education, 26, 434-461.

Buysse, V., Goldman, B.D., \& Skinner, M.L. (2002). Setting effects on friendship formation among young children with and without disabilities. Exceptional Children, 68, 503-517.

Buysse, V., Nabors, L., Skinner, D., \& Keyes, L. (1997). Playmate preferences and perceptions of individual differences among typically developing preschoolers. Early Child Development and Care, $131,1-18$.

Centre for Developmental Disability Studies. (2001). What is Developmental Disability. Retrieved August 12, 2003, from http://www.cdds.med.usyd.edu.au/html/what_is_dd.html

Chamberlain, B., Kasari, C., \& Rotherham-Fuller, E. (2007). Involvement or isolation? The social networks of children with autism in regular classrooms. Journal of Autism and Developmental Disorders, 37, 230-242.

Cleary, D.J., Ray, G.E., LoBello, S.G., \& Zachar, P. (2002). Children's perceptions of close peer relationships: Quality, congruence, and meta-perceptions. Child Study Journal, 32, 179-192.

Developmental Disabilities Assistance and Bill of Rights Act (2000). Retrieved August 11, 2003, from www.act.dhhs.gov/programs/add/DD-ACT2.htm

Coots, J.J., Bishop, K.D., Grenot-Scheyer, M., \& Falvey, M. A. (1995). Practices in general education: Past and present. In M.A. Falvey (Ed.), Inclusive and heterogeneous schooling (pp. 7-22). Baltimore: Paul H. Brookes.

Cuckle, P., \& Wilson, J. (2002). Social relationships and friendships among young people with Down's syndrome in secondary schools. British Journal of Special Education, 29, 66-71.

Evans, I.M., Salisbury, C.L., Palombaro, M.M., Berryman, J., \& Hollowood, T.M. (1992). Peer interactions and social acceptance of elementary-age children with severe disabilities in an inclusive school. Journal of the Association for Persons with Severe Handicaps, 17, 205-212.

Falvey, M.A. (1995). Services for students with disabilities: Past and present. In M.A. Falvey (Ed.), Inclusive and heterogeneous schooling (pp. 23-40). Baltimore: Paul H. Brookes.

Falvey, M.A., Givner, C.C., \& Kimm, C. (1995). What is an inclusive school? In R.A. Villa, \& J.S. Thousand (Eds.), Creating an inclusive school (pp. 1-15). Alexandria, VA: Association for Supervision and Curriculum Development.

Field, T. (1984). Play behaviors of handicapped children who have friends. In T. Field, J.L. Roopnarine, \& M. Segal (Eds.), Friendships in normal and handicapped children (pp. 153-162). Norwood, NJ: Ablex Publishing Corporation.

Freeman, S.F.N. (2000). Academic and social attainments of children with mental retardation in general education and special education settings. Remedial and Special Education, 21, 3-20.

Freeman, S.F.N., \& Kasari, C. (2002). Characteristics and qualities of the play dates of children with Down syndrome: Emerging or true friendships? American Journal on Mental Retardation, 107, 16-31.

Fujiki, M., Brinton, B., Hart, C.H., \& Fitzgerald, A.H. (1999). Peer acceptance and friendship in children with specific language impairment. Topics in Language Disorders, 19(2), 34-48.

Furman, W., \& Bierman, K.L. (1983). Developmental changes in young children's conceptions of friendship. Child Development, 54, 549-556.

Furman, W., \& Buhrmester, D. (1985). Children's perceptions of the personal relationships in their social networks. Developmental Psychology, 21, 1016-1024.

Gest, S.D., Graham-Bermann, S.A., \& Hartup, W.W. (2001). Peer experience: Common and unique features of number of friendships, social network centrality, and sociometric status. Social Development, 10(1), 23-40. 
Gottman, J.M. (1983). How children become friends. Monographs for the Society for Research in Child Development, 48(3, Serial No. 201).

Gresham, F.M., \& Elliott, S.N. (1990). Social Skills Rating System (SSRS). Circle Pines, Minnesota: American Guidance Service, Inc.

Guralnick, M.J. (1997). Peer social networks of young boys with developmental delays. American Journal on Mental Retardation, 101, 595-612.

Guralnick, M.J., \& Groom, J.M. (1988). Friendships of preschool children in mainstreamed playgroups. Developmental Psychology, 24, 595-604.

Hall, L.J. (1994). A descriptive assessment of social relationships in integrated classrooms. Journal of the Association for Persons with Severe Handicaps, 19, 302-313.

Hall, L.J., \& McGregor, J.A. (2000). A follow-up study of the relationships of children with disabilities in an inclusive school. Journal of Special Education, 34, 114-126.

Hall, L.J., \& Strickett, T. (2002). Peer relationships of preadolescent students with disabilities who attend a separate school. Education and Training in Mental Retardation and Developmental Disabilities, 37, 399-409.

Hanline, M.F. (1993). Inclusion of preschoolers with profound disabilities: An analysis of children's interactions. Journal of the Association for Persons with Severe Handicaps, 18, 28-35.

Heiman, T. (2000). Friendship quality among children in three educational settings. Journal of Intellectual \& Developmental Disability, 25, 1-12.

Hendrickson, J.M., Shokoohi-Yekta, M., Hamre-Nietupski, S., \& Gable, R.A. (1996). Middle and high school students' perceptions on being friends with peers with severe disabilities. Exceptional Children, 63, 19-28.

Hurley-Geffner, C.M. (1995). Friendships between children with and without developmental disabilities. In R.L. Koegel, \& L.K. Koegel (Eds.), Teaching children with autism (pp. 105-125). Baltimore: Paul H. Brookes.

Kennedy, C.H., \& Itkonen, T. (1994). Some effects of regular class participation on the social contacts and social networks of high school students with severe disabilities. Journal of the Association for Persons with Severe Handicaps, 19, 1-10.

Kerns, K.A. (2000). Types of preschool friendships. Personal Relationships, 7, 311-324.

Ladd, G.W., Kochenderfer, B.J., \& Coleman, C.C. (1996). Friendship quality as a predictor of young children's early school adjustment. Child Development, 67, 1103-1118.

Lee, S.H., Yoo, S.Y., \& Bak, S.H. (2003). Characteristics of friendships between children with and without mild disabilities. Education and Training in Mental Retardation and Developmental Disabilities, 38, 157-166.

Meyer, L.H., Minondo, S., Fisher, M., Larson, M.J., Dunmore, S., Black, J.W., et al. (1998). Frames of friendship: Social relationships among adolescents with diverse abilities. In L.H. Meyer, H.-S. Park, M. Grenot-Scheyer, I.S. Schwartz, \& B. Harry (Eds.), Making friends: The influences of culture and development (pp. 189-221). Baltimore: Paul H. Brookes.

Moore, C. (1998). Educating students with disabilities in general education classrooms: A summary of the research. Retrieved July 8, 2003, from http://interact.uoregon.edu/wrrc/AKInclusion.html

Murray-Seegert, C. (1989). Nasty girls, thugs, and humans like us: Social relations between severely disabled and non-disabled students in high school. Baltimore: Paul H. Brookes.

National Association of Developmental Disabilities Councils. (2003). What is a Developmental Disability? Retrieved August 11, 2003, from http://www.naddc.org/DD\%20Basics.htm

Newcomb, A.F., \& Bagwell, C.L. (1996). The developmental significance of children's friendship relations. In W.M. Bukowski, A.F. Newcomb, \& W.W. Hartup (Eds.), The company they keep: Friendship in childhood and adolescence (pp. 289-321). New York: Cambridge University Press.

Northern Territory Government. (2005). Central Region: Social-economic snapshot. Retrieved 23 July, 2007, from http://www.nt.gov.au/business/services.cfm?cat4id=14

Parker, J.G., \& Asher, S.R. (1993). Friendship and friendship quality in middle childhood: Links with peer group acceptance and feelings of loneliness and social dissatisfaction. Developmental Psychology, 29, 611-621. 
Ray, G.E., \& Cohen, R. (1996). Children's friendships: Expectations for prototypical versus actual best friends. Child Study Journal, 26, 209-227.

Rice, C. (2007). Prevalence of Autism Spectrum Disorders - Autism and Developmental Disabilities Monitoring Network, 14 Sites, United States, 2002. Retrieved 10 July, 2008, from http://www.cdc.gov/ MMWR/preview/mmwrhtml/ss5601a2.htm

Richardson, P., \& Schwartz, I.S. (1998). Making friends in preschool: Friendship patterns of young children with disabilities. In L.H. Meyer, H.-S. Park, M. Grenot-Scheyer, I.S. Schwartz, \& B. Harry (Eds.), Making friends: The influences of culture and development (pp. 65-80). Baltimore, MD: Paul H. Brookes.

Salisbury, C.L., \& Palombaro, M.M. (1998). Friends and acquaintances: Evolving relationships in an inclusive elementary school. In L.H. Meyer, H.-S. Park, M. Grenot-Scheyer, I.S. Schwartz, \& B. Harry (Eds.), Making friends: The influences of culture and development (pp. 81-104). Baltimore: Paul H. Brookes.

Searle, A. (1989). Cross-cultural perspectives on children's friendships in early childhood. Australian Journal of Early Childhood, 14(3), 35-38.

Siperstein, G.N., Leffert, J.S., \& Wenz-Gross, M. (1997). The quality of friendships between children with and without learning problems. American Journal on Mental Retardation, 102, 111-125.

Smith, A. (1998). Postmodern pedagogy: Toward a kinder, gentler nation? In L.H. Meyer, H.-S. Park, M. Grenot-Scheyer, I.S. Schwartz, \& B. Harry (Eds.), Making friends: The influences of culture and development (pp. xix-xxv). Baltimore: Paul H. Brookes.

Sparrow, S.S., Balla, D.A., \& Cicchetti, D.V. (1985). Vineland adaptive behavior scales: Classroom edition. Circle Pines, Minnesota: American Guidance Service, Inc.

Staub, D. (1998). Delicate threads: Friendships between children with and without disabilities. Bethesda, MD: Woodbine House.

Staub, D., Schwartz, I.S., Gallucci, C., \& Peck, C.A. (1994). Four portraits of friendship at an inclusive school. Journal of the Association for Persons with Severe Handicaps, 19, 314-325.

Strain, P.S. (1984). Social behavior patterns of nonhandicapped and developmentally disabled friend pairs in mainstream preschools. Analysis and Intervention in Developmental Disabilities, 4, 15-28.

Strully, J., \& Strully, C. (1985). Friendship and our children. Journal of the Association for Persons with Severe Disabilities, 10, 224-228.

TASH. (1999). TASH Resolution on Educating Young Children with Significant Disabilities. Retrieved July 8, 2003, from http://www.tash.org/resolutions/res02youngchild.htm

Turnbull, A.P., Blue-Banning, M., \& Pereira, L. (2000). Successful friendships of Hispanic children and youth with disabilities: An exploratory study. Mental Retardation, 38, 138-153.

Webster, A.A., \& Carter, M. (in press). Adaptation of an interview-based protocol to examine close relationships between children with developmental disabilities and peers. Australasian Journal of Special Education.

Westling, D. L., \& Fox, L. (2004). Teaching students with severe disabilities (3rd ed.). Upper Saddle River, NJ: Prentice Hall.

Wiener, J., \& Schneider, B.H. (2002). A multisource exploration of the friendship patterns of children with and without learning disabilities. Journal of Abnormal Child Psychology, 30, 127-141.

Zetlin, A.G., \& Murtaugh, M. (1988). Friendship patterns of mildly learning handicapped and nonhandicapped high school students. American Journal on Mental Retardation, 92, 447-454. 